\title{
Bifurcation Analysis of an Existing Mathematical Model Reveals Novel Treatment Strategies and Suggests Potential Cure for Type 1 Diabetes
}

\author{
Nielsen, Kenneth Hagde Mandrup; Ottesen, Johnny T.; Pociot, Flemming \\ Published in: \\ Mathematical Medicine and Biology (Print)
}

DOI:

10.1093/imammb/dqt006

Publication date:

2014

\section{Document Version}

Publisher's PDF, also known as Version of record

Citation for published version (APA):

Nielsen, K. H. M., Ottesen, J. T., \& Pociot, F. (2014). Bifurcation Analysis of an Existing Mathematical Model Reveals Novel Treatment Strategies and Suggests Potential Cure for Type 1 Diabetes. Mathematical Medicine and Biology (Print), 31(3), 205 - 225. https://doi.org/10.1093/imammb/dqt006

\section{General rights}

Copyright and moral rights for the publications made accessible in the public portal are retained by the authors and/or other copyright owners and it is a condition of accessing publications that users recognise and abide by the legal requirements associated with these rights.

- Users may download and print one copy of any publication from the public portal for the purpose of private study or research.

- You may not further distribute the material or use it for any profit-making activity or commercial gain.

- You may freely distribute the URL identifying the publication in the public portal.

Take down policy

If you believe that this document breaches copyright please contact rucforsk@kb.dk providing details, and we will remove access to the work immediately and investigate your claim. 
Mathematical Medicine and Biology (2014) 31, 205-225

doi:10.1093/imammb/dqt006

Advance Access publication on April 24, 2013

\title{
Bifurcation analysis of an existing mathematical model reveals novel treatment strategies and suggests potential cure for type 1 diabetes
}

\author{
KenNeth H. M. NielseN* \\ Department of Science, Systems, and Models, Roskilde University, Universitetsvej 1, \\ Building 27, 4000 Roskilde, Denmark \\ *Corresponding author: khmn@ ruc.dk \\ FLEMming M. Pociot \\ Glostrup Research Institute, Glostrup Hospital, Nordre Ringvej 69, Entrance North, \\ 2600 Glostrup, Denmark \\ AND \\ JOHNNY T. OTTESEN \\ Department of Science, Systems, and Models, Roskilde University, Universitetsvej 1, \\ Building 27, 4000 Roskilde, Denmark
}

[Received on 22 May 2012; revised on 3 February 2013; accepted on 26 March 2013]

Type 1 diabetes is a disease with serious personal and socioeconomic consequences that has attracted the attention of modellers recently. But as models of this disease tend to be complicated, there has been only limited mathematical analysis to date. Here we address this problem by providing a bifurcation analysis of a previously published mathematical model for the early stages of type 1 diabetes in diabetesprone NOD mice, which is based on the data available in the literature. We also show positivity and the existence of a family of attracting trapping regions in the positive 5D cone, converging towards a smaller trapping region, which is the intersection over the family. All these trapping regions are compact sets, and thus, practical weak persistence is guaranteed. We conclude our analysis by proposing 4 novel treatment strategies: increasing the phagocytic ability of resting macrophages or activated macrophages, increasing the phagocytic ability of resting and activated macrophages simultaneously and lastly, adding additional macrophages to the site of inflammation. The latter seems counter-intuitive at first glance, but nevertheless it appears to be the most promising, as evidenced by recent results.

Keywords: bifurcation analysis; codimension 1; codimension 2; mathematical modelling; NOD; type 1 diabetes (IDDM); model-guided experiments; treatment; cure; attracting trapping region.

\section{Introduction}

The consensus of today is that type 1 diabetes (T1D) is caused by a selective depletion of the insulinproducing pancreatic $\beta$ cells as a result of their falling prey to an autoimmune reaction (Höglund et al., 1999). In humans it is putative that T1D is triggered by external factors (Skyler, 2007) in genetically susceptible individuals (Maier \& Wicker, 2005). As the $\beta$ cells are removed, the body becomes unable to maintain the blood-glucose homeostasis, and eventually hyperglycemic T1D ensues. Without daily subcutaneous injections of insulin the disease is fatal (Lo \& Clare-Salzler, 2006). Insulin, however, only treats the symptoms, not the cause of the sickness itself. T1D affects $0.5 \%$ of people in the developed countries, in addition $5-15 \%$ of those diagnosed with type 2 diabetes (T2D) are thought 
to have T1D—not T2D (Mathis et al., 2001). Furthermore, the number of people with T1D (and T2D) is expected to soar during the next 20 years (Wild et al., 2004). This makes the search for viable methods of T1D treatment ever more important.

Diabetes, especially T1D, is hard to study in vivo in humans (Sparre et al., 2005), which is one of the reasons why mathematical modelling has found its way into this field of research, where it, in conjunction with data obtained from animal models of diabetes and in vitro experiments, aids in understanding various aspects of the disease. The focus of this paper is one such mathematical model proposed by Marée et al. (2006), which we have dubbed the DuCa model, as it is the result of a collaboration between Dutch and Canadian researchers, and the biological implications of the bifurcation analysis of the model. The DuCa model deals with the stages of T1D that are prior to infiltration by T cells, and derives some of its mathematical background from the Copenhagen model, which was proposed by Freiesleben De Blasio et al. (1999). The key conclusion is that a chronic autoimmune process is established before T cells become involved (Marée et al., 2006). To our knowledge, the authors of the DuCa model have not performed a bifurcation analysis before, nor have they established practical weak persistence as the one we present here.

The main result of our analysis is four novel treatment strategies. The first three arise from our codimension 1 and 2 analyses and are intuitively straightforward. The first two methods consist of increasing the phagocytic ability of either the resting or the activated macrophages. The third is increasing the phagocytosis rates of the resting as well as the activated macrophages. The fourth treatment strategy is more counter-intuitive. It entails boosting the immune response by adding additional macrophages to the site of inflammation. This is contrary to the idea that has generally been pursued, which involves decreasing the immune response.

The organization of this paper is as follows: in Section 2 a well-accepted hypothesis of an early phase of T1D is presented. The underlying mechanisms are unveiled and two different mouse strains are characterized. The mechanisms explained in Section 2 give rise to the mathematical model by Marée et al. (2006), which is provided in Section 3. Section 4 provides the existence and uniqueness of solutions and the construction of a family of attracting trapping regions. It is also shown that the intersection of these is itself a trapping region. Section 5 covers two codimension 1 analyses and in Section 6 aspects of a codimension 2 analysis, which has physiological implications, are discussed. Section 7 introduces an important feature of the system and some results that are important when interpreted within the biological setting of the DuCa model. In Section 8 we gather the results of the bifurcation analysis and interpret them in terms of prospective treatment strategies. Finally Section 9 contains a discussion of the results and proposes different ways of testing them.

\section{Biological background}

The background of the model presented in Marée et al. (2006) has to do with the way macrophages, a type of immune cell, respond to $\beta$ cells that are terminating through either apoptosis or necrosis. Apoptosis is commonly described as 'programmed cell death'. It entails an organized shutting-down of the cell whereby the cell shrinks, and no or little cell-debris is spilt (Steer et al., 2006). The result of this 'quiet' death is that no inflammation ensues. Necrosis, on the other hand, is associated with inflammation, and entails loss of cell-membrane integrity, which implies spillage of cell-debris (Zong $\&$ Thompson, 2006). The difference between an apoptotic and a necrotic $\beta$ cell plays an integral part in the model presented in Marée et al. (2006).

Full-blown T1D is preceded by a long period of decline in $\beta$ cell mass (Skyler, 2007). As the $\beta$ cells start terminating, resting macrophages will try to clear them through a process called phagocytosis. 
When macrophages phagocytize apoptotic cells they become activated, which in healthy mouse-strains up-regulates their phagocytic ability. However, if the macrophages are unable to clear the $\beta$ cells fast enough, the $\beta$ cells become secondary necrotic (Marée et al., 2006; Zong \& Thompson, 2006). When an activated macrophage phagocytizes a necrotic $\beta$ cell, it elicits an inflammatory response that involves secretion of a number of signal proteins called cytokines (Marée et al., 2006; Alberts et al., 2008). In T1D some of the cytokines are cytotoxic to $\beta$ cells and will induce apoptosis or necrosis in otherwise healthy $\beta$ cells (Stoffels et al., 2004; Steer et al., 2006), thus adding to the amount of $\beta$ cells that needs clearing.

Marée et al. (2005) investigated the phagocytic ability of macrophages from the diabetes-prone NOD mouse-strain and the Balc/c-strain which does not (in general) develop diabetes. It was concluded that macrophages from Balb/c mouse strain, are generally more efficient at phagocytizing apoptotic $\beta$ cells than NOD macrophages - the resting Balb/c macrophages were twice as effective at phagocytizing than the their NOD counterparts. Furthermore, they found that Balb/c macrophages undergo an activation step after they have engulfed the first apoptotic $\beta$ cell. After the activation step, their phagocytosis rate increases. In NOD mice no activation step was observed, i.e. the NOD macrophages do not become more efficient at phagocytizing after engulfment of an apoptotic $\beta$ cell-the activated Balb/c macrophages were found to phagocytize 5 times as fast as the 'activated' NOD macrophages. These results were refined and confirmed by Marée et al. (2008).

In addition to these results, it has been shown that in rodents a large number of pancreatic $\beta$ cells undergo apoptosis, as part of a pancreatic remodelling at the neonatal stage (Scaglia et al., 1997; Trudeau et al., 2000; Mathis et al., 2001). Similar results have been reported in pigs (Bock et al., 2003) and in humans (Kassem et al., 2000). In the following we will refer to this event as the apoptotic wave. These findings led to the hypothesis that the reason why T1D is more prevalent in NOD mice (relative to other mouse strains) is the poor phagocytosis rate of their macrophages (e.g. Trudeau et al., 2000; Mathis et al., 2001). The greater phagocytosis rate in Balb/c mice implies that the macrophages are able to accommodate the increased amount of apoptotic $\beta$ cells during the apoptotic wave, whereas this is not the case in NOD mice. Here, so many of the apoptotic $\beta$ cells are left uncleared long enough for the cells to become necrotic, so that the pool of necrotic cells induces a production of cytokines large enough to maintain a depletion of healthy $\beta$ cells; i.e. initiates chronic inflammation.

In summary the biological reasoning behind the model presented by Marée et al. (2006) can be summarized as follows: an apoptotic wave occurs, which causes an accumulation of apoptotic $\beta$ cells, and as the resting macrophages start to phagocytize the $\beta$ cells they become activated. In NOD mice, in particular, a portion of the apoptotic $\beta$ cells are left uncleared long enough for them to become necrotic. When the activated macrophages engulf and phagocytize the necrotic $\beta$ cells, they secrete different cytokines, of which some are cytotoxic to the healthy $\beta$ cells. The cytotoxic cytokines cause the healthy $\beta$ cells to become apoptotic, thus adding to the accumulation of apoptotic $\beta$ cells.

\section{Mathematical model}

For the sake of readability of the following chapters, we restate the DuCa model here; parameters and model are identical to Marée et al. (2006) save for a few slight changes: compared with the original model, we have set $J=a, e_{1}=e_{2}=e$ and $g=f_{1}$. (Asymmetry in the competition terms, i.e. having $e_{1} \neq e_{2}$ leads to competitive exclusion De Boer \& Perelson, 1994.) The model consists of a system of 5 coupled ODEs that describe the change in concentration of resting macrophages, $M$, activated macrophages, $M_{a}$, apoptotic $\beta$ cells, $B_{a}$, necrotic $\beta$ cells, $B_{n}$, and cytokines, $C$. The equation describing the change in concentration of apoptotic $\beta$ cells is non-autonomous due to the apoptotic wave, 
represented here by $W(t)$.

$$
\begin{aligned}
\frac{\mathrm{d} M}{\mathrm{~d} t} & =a+(k+b) M_{a}-c M-f_{1} M B_{a}-e M\left(M+M_{a}\right), \\
\frac{\mathrm{d} M_{a}}{\mathrm{~d} t} & =f_{1} M B_{a}-k M_{a}-e M_{a}\left(M+M_{a}\right), \\
\frac{\mathrm{d} B_{a}}{\mathrm{~d} t} & =W(t)+\frac{A_{\max } C}{k_{c}+C}-f_{1} M B_{a}-f_{2} M_{a} B_{a}-d B_{a}, \\
\frac{\mathrm{d} B_{n}}{\mathrm{~d} t} & =\mathrm{d} B_{a}-f_{1} M B_{n}-f_{2} M_{a} B_{n} \\
\frac{\mathrm{d} C}{\mathrm{~d} t} & =\alpha B_{n} M_{a}-\delta C .
\end{aligned}
$$

For an illustration of the model and a discussion of parameters, we refer the reader to Marée et al. (2006). Table A1 given in Appendix A provides an overview of the values, units and meaning of the parameters. Parameters discussed from hereon have the units provided in Table A1.

\section{Mathematical analysis: existence of solution and trapping regions}

In the following sections we will use the notation $\mathbf{x}=\left(x_{1}, x_{2}, x_{3}, x_{4}, x_{5}\right)$ for $\left(M, M_{a}, B_{a}, B_{n}, C\right)$.

The existence and uniqueness of solutions to initial value problems of the system follows: since the apoptotic wave is modelled by a non-autonomous function solution curves may cross in phase space. This can be remedied by the change of variables $t=y$ and adding the equation $\dot{y}=1$, with initial value $y(0)=0$, to the system. By doing so we obtain a system that is Lipschitz continuous in $\left(\mathbb{R}_{+} \cup\right.$ $\{0\})^{6}$, since all equations are continuously differentiable in this domain, which guarantees existence and uniqueness (of the 6D system).

\subsection{Trapping regions}

Besides existence and uniqueness, it is always a priority to establish whether one's solutions are wellbehaved in the physiological sense; i.e. if solutions starting in the positive cone stay in the positive cone; i.e. $x_{i}(t) \geqslant 0$, for all $i=1, \ldots, 5$ and $t>0$, if $x_{i}(0) \geqslant 0$, for all $i=1, \ldots, 5$, and solutions are uniformly bounded; i.e. there exists a constant $\bar{x}_{i}>0$ such that $x_{i}(t) \leqslant \bar{x}_{i}$ for all $t>0$ and $i=1, \ldots, 5$. Equivalently, this means that $\underline{x}_{i}$ and $\bar{x}_{i}$ exist such that $0 \leqslant \underline{x}_{i} \leqslant \liminf _{t \rightarrow \infty} x_{i}(t) \leqslant \lim \sup _{t \rightarrow \infty} x_{i}(t) \leqslant \bar{x}_{i}, i=1, \ldots, 5$, which we refer to as practical weak persistence; lim inf and lim sup denote limit inferior and superior, respectively. This generally follows if it is possible to construct a trapping region, $\mathfrak{T} \mathfrak{R}$, which we define as an outflow-closed region; a region in phase space that fulfill that once in this region the solution cannot leave it. In our case the trapping region is in $\left(\mathbb{R}_{+} \cup 0\right)^{5}$. Obviously, we cannot extend the trapping region to the $6 \mathrm{D}$ system due to the solution for $y$. We start by showing positivity, i.e. $x_{i}(t) \geqslant 0$ for all $t>0$ and $i=1, \ldots, 5$, if $x_{i}(0) \geqslant 0$ for all $i=1, \ldots, 5$.

Observe that (3.1-3.5) are of the form

$$
\dot{x}_{i}=f_{i}(\mathbf{x})-g_{i}(\mathbf{x}) x_{i}
$$

where $f_{i} \geqslant 0$ does not depend on $x_{i}, f_{1}(\mathbf{x})>0$ and $g_{i} \geqslant 0$. 
To ensure that the flow is not pointing out of $\left(\mathbb{R}_{+} \cup\{0\}\right)^{5}$ on any of the hyperplanes defined by $x_{i}=0$ for $i=1, \ldots, 5$, we look at the sign of $\dot{x}_{i}$ for $x_{i}=0$ with the other variables $\geqslant 0$. From (4.1) we see that $\dot{x}_{1}>0$ for $x_{1}=0$ and $\dot{x}_{i} \geqslant 0$, for $x_{i}=0, i=2, \ldots, 5$. So the flow is contained in $\left(\mathbb{R}_{+} \cup\{0\}\right)^{5}$ by the positive hyperplanes $x_{i}=0, i=1, \ldots, 5$. Hence, the flow will not leave the positive orthant once it enters it.

Next we establish that the concentrations cannot grow indefinitely by constructing a trapping region $\mathfrak{T R}=\left[\underline{x}_{1}, \bar{x}_{1}\right] \times\left[\underline{x}_{2}, \bar{x}_{2}\right] \times\left[\underline{x}_{3}, \bar{x}_{3}\right] \times\left[\underline{x}_{4}, \bar{x}_{4}\right] \times\left[\underline{x}_{5}, \bar{x}_{5}\right]$ for some constants $\underline{x}_{i}, \bar{x}_{i} \in \mathbb{R}_{+} \cup\{0\}$ with $\underline{x}_{i}<\bar{x}_{i}$, and $i=1, \ldots, 5$. From the foregoing discussion we see that candidates for lower bounds, $\underline{x}_{i}$, may be chosen as zero. However, as we will see, we need to establish a nonzero lower bound for at least one variable. As for the upper bounds, we wish to show that the derivatives become negative if the variables are allowed to become sufficiently large.

To simplify things, we start by defining $X_{m}=x_{1}+x_{2}$, where the subscript, $m$, denotes macrophages, (i.e. $X_{m}=M+M_{a}$ ), then $\dot{X}_{m}=\dot{x}_{1}+\dot{x}_{2}$. This gives us

$$
a-c X_{m}-e X_{m}^{2}<\dot{X}_{m} \leqslant a+b X_{m}-e X_{m}^{2} .
$$

Thus for

$$
X_{m} \leqslant \underline{X}_{m}:=\sqrt{\frac{c^{2}}{4 e^{2}}+\frac{a}{e}}-\frac{c}{2 e},
$$

we get $\dot{X}_{m} \geqslant 0$ with equality if, and only if, $X_{m}=\underline{X}_{m} \cdot \underline{X}_{m}>0$ will prove important shortly. For

$$
X_{m} \geqslant \bar{X}_{m}:=\frac{b}{2 e}+\sqrt{\frac{b^{2}}{4 e^{2}}+\frac{a}{e}},
$$

we have $\dot{X}_{m} \leqslant 0$, with equality if, and only if, $X_{m}=\bar{X}_{m}$. For the construction of the trapping region, define $R_{12}:=\left\{\left(x_{1}, x_{2}\right) \in \mathbb{R}^{2}: x_{1} \geqslant 0, x_{2} \geqslant 0, \underline{X}_{m} \leqslant x_{1}+x_{2}<\bar{X}_{m}\right\}$. Next set $X_{b}=x_{3}+x_{4}$, where the subscript $b$ denotes $\beta$-cells (i.e. $X_{b}=B_{a}+B_{n}$ ), then

$$
\dot{X}_{b}=W(t)+\frac{A_{\max } x_{5}}{k_{c}+x_{5}}-\left(f_{1} x_{1}+f_{2} x_{2}\right)\left(X_{b}\right) .
$$

The apoptotic wave is assumed to peak after 9 days, so set $K_{\max }:=W(9)+A_{\max }$, then

$$
\dot{X}_{b}<K_{\max }-\min \left\{f_{1}, f_{2}\right\} X_{m} X_{b}<K_{\max }-\min \left\{f_{1}, f_{2}\right\} \underline{X}_{m} X_{b}
$$

where $\min \left\{f_{1}, f_{2}\right\}$ indicates the minimum value of the two phagocytosis rates. Set $\bar{X}_{b}:=$ $\left(K_{\max } / \min \left\{f_{1}, f_{2}\right\} \underline{X}_{m}\right)$, then $\dot{X}_{b}<0$ for $X_{b}>\bar{X}_{b}$; the construction of $\bar{X}_{b}$ was the reason we needed $\underline{X}_{m}>0$. Define $R_{34}:=\left\{\left(x_{3}, x_{4}\right) \in \mathbb{R}^{2}: x_{3} \geqslant 0, x_{4} \geqslant 0,0 \leqslant x_{3}+x_{4}<\bar{X}_{b}\right\}$. Figure 1 provides an illustration of the sets $R_{12}$ and $R_{34}$. Bold lines are the boundaries of the sets, with dashed lines indicating the upper boundaries, which are not included in the sets. If the concentrations of resting and activated macrophages start in $R_{12}$, they will not leave it. A similar statement holds for apoptotic and necrotic $\beta$ cells in $R_{34}$.

Finally, if $x_{5}>(\alpha / \delta) \bar{X}_{m} \bar{X}_{b}$ then $\dot{x}_{5}<0$ so set $\bar{x}_{5}:=(\alpha / \delta) \bar{X}_{m} \bar{X}_{b}$, and define $R_{5}:=\left[0, \bar{x}_{5}\right]$. Hence, the Cartesian product, $R_{12} \times R_{34} \times R_{5}$ of $R_{12}, R_{34}$, and $R_{5}$ defines the trapping region $\mathfrak{T} \Re$. Please notice that $\mathfrak{T} \mathfrak{R}$ is not a minimal trapping region: by considering each of the variables by themselves a smaller trapping region can be obtained. However, that requires more work, and any trapping region will suffice.

Next we would have liked to show that for initial values in the positive orthant but not in $\mathfrak{T} \Re, x_{i}(t)$ enters the trapping region in finite time. This would imply that $\mathfrak{T} \mathfrak{R}$ is an attracting trapping region. 

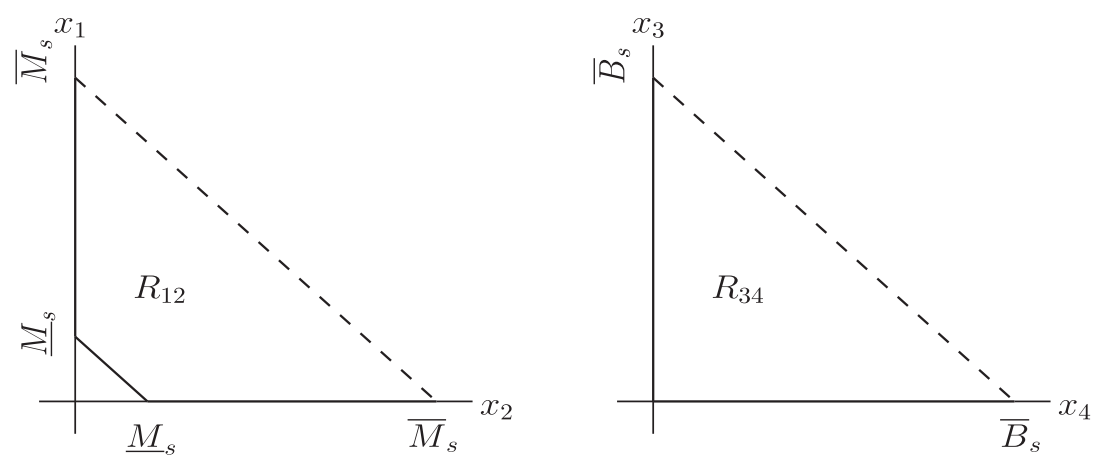

FIG. 1. Graphical illustration of the sets $R_{12}$ and $R_{34}$. Bold lines indicate boundaries of the sets. Dashed lines are the upper boundaries that are not included in the sets.

However, since it is possible that $\dot{x}_{i}=0$ for $x_{i}=\bar{x}_{i}$, for some $i$, we cannot prove this, but we can come arbitrarily close to the result. For this purpose we construct a family of trapping regions, $\mathfrak{T} \mathfrak{R}$, and prove that these are attracting and that the intersection of these gives $\mathfrak{T} R$.

First define

$$
\begin{aligned}
\tilde{R}_{12} & :=\left\{\left(x_{1}, x_{2}\right): x_{1} \geqslant 0, x_{2} \geqslant 0, \underline{X}_{m} \leqslant x_{1}+x_{2}<\tilde{X}_{m}\right\} \quad \text { with } \tilde{X}_{m}>\bar{X}_{m}, \\
\tilde{R}_{34} & :=\left\{\left(x_{3}, x_{4}\right): x_{3} \geqslant 0, x_{4} \geqslant 0,0 \leqslant x_{3}+x_{4}<\tilde{X}_{b}\right\} \quad \text { with } \tilde{X}_{b}>\bar{X}_{b}, \\
\tilde{R}_{5} & :=\left[0, \tilde{x}_{5}\right] \quad \text { with } \tilde{x}_{5}>\bar{x}_{5} .
\end{aligned}
$$

Then going through a construction similar to that for $\mathfrak{T} \mathfrak{R}$, it follows that $\widetilde{\mathfrak{T} R}=\tilde{R}_{12} \times \tilde{R}_{34} \times \tilde{R}_{5}$ is also a trapping region. Hence the flow becomes strictly negative on the corresponding upper boundaries of $\widetilde{T} \Re$. Now, for any $\epsilon>0$, let $\tilde{R}_{12}^{\epsilon}$ be $\tilde{R}_{12}$ with the substitution $\tilde{X}_{m}=\bar{X}_{m}+\epsilon$, and construct $\tilde{R}_{34}^{\epsilon}$ in an analogous way. This gives us $\tilde{x}_{5}=\alpha / \delta\left(\bar{X}_{m}+\epsilon\right)\left(\bar{X}_{b}+\epsilon\right)$. Define $\mathfrak{T R}^{\epsilon}=\tilde{R}_{12}^{\epsilon} \times \tilde{R}_{34}^{\epsilon} \times \tilde{R}_{5}^{\epsilon}$, then all $\mathfrak{T R}^{\epsilon}$ 's are also trapping regions, of the form $\widetilde{\mathfrak{T} R}$, for any $\epsilon>0$, and $\mathfrak{T} \mathfrak{R}=\bigcap_{\epsilon>0} \mathfrak{T} \mathfrak{R}^{\epsilon}$, i.e. $\mathfrak{T} \mathfrak{R}^{\epsilon} \rightarrow \mathfrak{T} \mathfrak{R}$ for $\epsilon \rightarrow 0$.

Proposition 4.1 For any $\epsilon>0, \mathfrak{T} \mathfrak{R}^{\epsilon}$ is a trapping region for the ODE system given in (3.1-3.5), with $\mathfrak{T R}=\bigcap_{\epsilon>0} \mathfrak{T} \mathfrak{R}^{\epsilon}$.

Next we show that the trapping regions $\mathfrak{T} \mathfrak{R}^{\epsilon}$ are attracting for every $\epsilon>0$, and any initial condition in the positive orthant, but not in $\mathfrak{T} \mathfrak{R}^{\epsilon}$, enters into it in finite time.

Proposition 4.2 Any point in $\left(\mathbb{R}_{+} \cup\{0\}\right)^{5} \backslash \mathfrak{T} \mathfrak{R}^{\epsilon}$ is attracted to $\mathfrak{T} \mathfrak{R}^{\epsilon}$, and it enters the trapping region in finite time. Hence, $\mathfrak{T} \mathfrak{R}^{\epsilon}$ is an attracting trapping region in the positive cone for any $\epsilon>0$.

Thus we need to show that if we choose $\mathbf{x}(0) \in \mathbb{R}_{+}^{5} \backslash \mathfrak{T} \mathfrak{R}^{\epsilon}$ then at some time, $T_{i}, x_{i}(t)$, has entered $\operatorname{proj}_{i}\left(\mathfrak{T} \mathfrak{R}^{\epsilon}\right)$, where $i=1, \ldots, 5$, and $\operatorname{proj}_{i}\left(\mathfrak{T} \mathfrak{R}^{\epsilon}\right)$ is the projection of $\mathfrak{T} \mathfrak{R}^{\epsilon}$ onto the $x_{i}$-axis-e.g. $\operatorname{proj}_{5}\left(\mathfrak{T} \mathfrak{R}^{\epsilon}\right)=\left[0, \tilde{x}_{5}\right]$-and for $T=\sum_{i=1}^{5} T_{i}$, all the $x_{i}(t)$ 's enter $\operatorname{proj}_{i}\left(\mathfrak{T} \mathfrak{R}^{\epsilon}\right)$.

Proof. Given any $\epsilon>0$ and $\mathbf{x}(0) \in\left(\mathbb{R}_{+} \cup\{0\}\right)^{5} \backslash \mathfrak{T} \mathfrak{R}_{\epsilon}$. Then $x_{i}(0) \notin \operatorname{proj}_{i}\left(\mathfrak{T} \mathfrak{R}_{\epsilon}\right)$ for some $i=1, \ldots, 5$. If $x_{1}(0) \in \operatorname{proj}_{1}\left(\mathfrak{T R}^{\epsilon}\right)$, we put $T_{1}=0$. If $x_{1}(0)>\tilde{X}_{m}$, we have that $\inf _{\left[\tilde{X}_{m}, x_{1}(0)\right]}\left\{\dot{x}_{1}\right\}=\min _{\left[\tilde{X}_{m}, x_{1}(0)\right]}\left\{\dot{x}_{1}\right\}:=$ $m_{1}<0$ is finite, since $\dot{x}_{1}$ is continuous and negative. Thus, $1 / \dot{x}_{1} \leqslant 1 / m_{1}<0$ on $\left[\tilde{X}_{m}, x_{1}(0)\right]$, and the time 
it takes $x_{1}$ to enter into $\operatorname{proj}_{1}\left(\mathfrak{T} \mathfrak{R}^{\epsilon}\right)$ is

$$
\int_{x_{1}(0)}^{\tilde{X}_{m}} \frac{\mathrm{d} x_{1}}{\dot{x}_{1}} \leqslant \int_{x_{1}(0)}^{\tilde{X}_{m}} \frac{\mathrm{d} x_{1}}{m_{1}}=\left(\tilde{X}_{m}-x_{1}(0)\right) / m_{1}:=T_{1} .
$$

If $x_{1}(0)<\underline{X}_{m}$, then $\min _{\left[x_{1}(0), \underline{X}_{m}\right]}\left\{\dot{x}_{1}\right\}:=m_{1}>0$ is finite. Thus, $1 / m_{1} \geqslant 1 / \dot{x}_{1}>0$ on $\left[x_{1}(0), \underline{X}_{m}\right]$, and the time it takes $x_{1}$ to enter into $\operatorname{proj}_{1}\left(\mathfrak{T} \mathfrak{R}^{\epsilon}\right)$ is

$$
\int_{x_{1}(0)}^{\underline{X}_{m}} \frac{\mathrm{d} x_{1}}{\dot{x}_{1}} \leqslant \int_{x_{1}(0)}^{\underline{X}_{m}} \frac{\mathrm{d} x_{1}}{m_{1}}=\left(\underline{X}_{m}-x_{1}(0)\right) / m_{1}:=T_{1} .
$$

During the timespan $T_{1}, \mathbf{x}$ travels from $\mathbf{x}(0)$ to $\mathbf{x}\left(T_{1}\right)$ such that $x_{1}\left(T_{1}\right) \in \operatorname{proj}_{1}\left(\mathfrak{T} \mathfrak{R}^{\epsilon}\right)$. Thereafter, $x_{1}(t)$ is trapped in $\operatorname{proj}_{1}\left(\mathfrak{T} \mathfrak{R}^{\epsilon}\right)$ for all future times $t>T_{1}$.

Now if $x_{3}\left(T_{1}\right) \in \operatorname{proj}_{3}\left(\mathfrak{T} \mathfrak{R}^{\epsilon}\right)$, put $T_{3}=0$, else $m_{3}=\min _{\left[\tilde{X}_{b}, x_{3}\left(T_{1}\right)\right]}\left\{\dot{x}_{3}\right\}=\inf _{\left[\tilde{X}_{b}, x_{3}\left(T_{1}\right)\right]}\left\{\dot{x}_{3}\right\}<0$ is finite. As before the time it takes $x_{3}\left(T_{1}\right)$ to enter into $\operatorname{proj}_{3}\left(\mathfrak{T} \mathfrak{R}^{\epsilon}\right)$ is less than or equal to $T_{3}=\left(\tilde{X}_{b}-x_{3}\left(T_{1}\right)\right) / m_{3}$. For all future times $x_{3}(t)$ is trapped in $\operatorname{proj}_{3}\left(\mathfrak{T} \mathfrak{R}^{\epsilon}\right)$.

Similarly, we construct finite positive times $T_{2}, T_{4}, T_{5}$ such that $x_{2}\left(T_{1}+T_{3}+T_{2}\right) \in \operatorname{proj}_{2}\left(\mathfrak{T} \mathfrak{R}^{\epsilon}\right)$, and $x_{2}(t)$ is trapped there for all future times, $x_{4}\left(T_{1}+T_{3}+T_{2}+T_{4}\right) \in \operatorname{proj}_{4}\left(\mathfrak{T} \mathfrak{R}^{\epsilon}\right)$, and $x_{4}(t)$ is trapped there for all future times, and, finally $x_{5}\left(T_{1}+T_{3}+T_{2}+T_{4}+T_{5}\right) \in \operatorname{proj}_{5}\left(\mathfrak{T} \mathfrak{R}^{\epsilon}\right)$, and $x_{5}(t)$ is trapped there for all future times. Hence, letting $T=\sum_{i=1}^{5} T_{i}$, it follows that $\mathbf{x}(T) \in \mathfrak{T} \mathfrak{R}^{\epsilon}$ and stays there for all future times $t>T$.

By this construction $\mathfrak{T} \mathfrak{R}^{\epsilon}$ is an attracting trapping region.

\section{Bifurcation analysis}

The defining assumption of Marée et al. (2006) is that the difference in the phagocytic ability between NOD and Balb/c mice, alone, is responsible for their distinct fates (Marée et al., 2006). In other words, depending on how we choose the phagocytosis rates the model should tend either to a stable state that corresponds to a disease state of chronic inflammation, which we denote by $\mathbf{x}_{\mathrm{DS}}^{*}$, or to a stable state that corresponds to a healthy, or non-inflammatory, state, $\mathbf{x}_{H}^{*}$, as $t \rightarrow \infty$. This makes the phagocytosis parameters $\left(f_{1}, f_{2}\right)$ the natural bifurcation parameters. For this reason we perform a numerical codimension 1 analysis using $f_{1}$ and $f_{2}$ as bifurcation parameters. Here, we will only deal with the analyses based on NOD parameters as these are the most interesting from a medical point of view - the Balb/c mouse does not become diabetic within the window of parameters studied here.

In the process of producing bifurcation diagrams we used an autonomous version of the DuCa model - the model without the $W(t)$-term-in the bifurcation continuation scheme. We did so because we wanted the 'full picture'. Since the apoptotic wave is ultimately responsible for the long-term behaviour of the system, e.g. pushing the system into the domain where $\mathbf{x}_{\mathrm{DS}}^{*}$ is an attracting fixed point, we applied proper initial conditions in lieu of the wave. In other words, we replaced the wave with an initial pool of apoptotic $\beta$ cells, $B_{a}(0)$, when doing continuations of the $\mathbf{x}_{\mathrm{DS}}^{*}$ and set $B_{a}(0)=0$ when doing a continuation of $\mathbf{x}_{H}^{*}$. The initial pool was chosen based on the procedure described below.

We used a Newton-Raphson method to produce the bifurcation diagrams. The method requires initial guesses from which to start its iterative process. To this end we ran simulations of the nonautonomous DuCa model for different values of $f_{1}$ (or $f_{2}$ ) until the concentrations had stabilized sufficiently. Then we used these concentration values as $x_{i}(0)$ s, i.e. initial guesses, for our bifurcation 

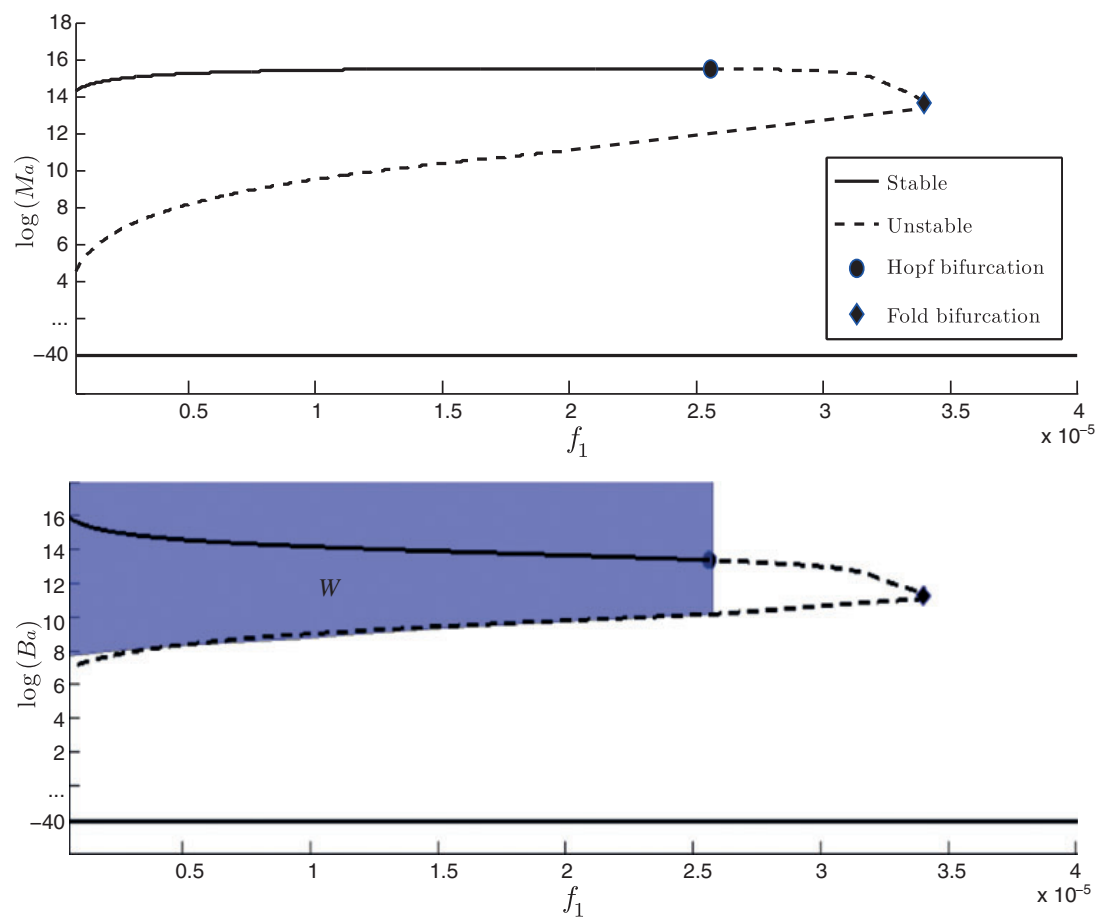

FIG. 2. Bifurcation diagrams showing $\log _{e}\left(M_{a}\right)$ (top) and $\log _{e}\left(B_{a}\right)$ (bottom) versus the phagocytosis rate of resting macrophages, $f_{1}$. All other parameters are as given in Table A1. Notice that the y-axes have been modified to facilitate the overview. The three dots on the y-axes indicate the part of the y-range that has been removed. Solid lines consist of stable fixed points. Dashed lines indicate unstable fixed points. The uppermost line is the disease state, and the lowest line is the disease-free state (in both diagrams). They are separated by a state of low-level inflammation, which is unstable. Two bifurcations occur as $f_{1}$ is varied. At $f_{1} \approx 2.57 \times 10^{-5}$ a Hopf bifurcation (HB) occurs (indicated by filled circle), and a fold bifurcation happens at $f_{1} \approx 3.45 \times 10^{-5}$ (indicated by filled diamond). The system is bistable for $f_{1}$ values lower than the Hopf point.

continuation scheme. (PyDSTool Clewley et al., 2007 and XPPAUT Ermentrout, 1987 produce similar diagrams, but struggle to produce diagrams with $f_{1}$ as the bifurcation parameter.)

Using $f_{1}$ as the bifurcation parameter: Figure 2 shows a bifurcation diagram with the $\log _{e^{-}}$ concentration of activated macrophages (top) and apoptotic $\beta$ cells (bottom) plotted against $f_{1}$ - the phagocytosis rate of resting macrophages; all other parameters are as in Table A1. The y-axes have been modified to reflect that we are plotting the logarithm of the concentration of activated macrophages (and apoptotic $\beta$ cells). Thus, the solid line through $\log \left(M_{a}\right)=-40$ (and $\log \left(B_{a}\right)=-40$ ) is the disease-free state, i.e. where $M_{a}=0$ (and $B_{a}=0$ ), and ' . .' indicates the part of the y-axes we have removed. Dashed lines represent unstable fixed points, while solid lines consist of stable fixed points. For $f_{1}$ lower than $\approx 2.57 \times 10^{-5}$ the system is bistable, meaning that the diseased and healthy state are both stable, or attracting. The dashed line separating the two states consists of unstable states of low-level inflammation and serves as a threshold between health and inflammation. When the phagocytosis rate of resting macrophages reaches $\approx 2.57 \times 10^{-5}$, the upper full line changes into a dashed line, indicating that the disease state changes stability. Eigenvalue inspection (not shown) reveals that at this approximate value of $f_{1}$, the real value of two complex eigenvalues changes sign from negative to positive. Thus, we are dealing with a HB. Simulations with $f_{1}$ values to the left of, and initial conditions close to, the HB 
point exhibit spirals that tend to the stable disease state (damped oscillations), while simulations with $f_{1}$ values just to the right of the HB point, and initial conditions perturbed slightly from the disease state, spiral away from the disease state, and eventually end up in the healthy state.

Following this the, now unstable, disease state descends with increasing $f_{1}$ until it amalgamates with the line of, unstable, low-level inflammation from beneath at $f_{1} \approx 3.45 \times 10^{-5}$ in a fold bifurcation. Thus for $f_{1}>2.57 \times 10^{-5}$, there are two unstable branches and one stable branch-the healthy state. As a point of reference for these values of $f_{1}$, we remark that the $f_{1}$ value of Balb/c macrophages that was used in Marée et al. (2006) is $f_{1}=2 \times 10^{-5}$.

Though we have not been able to determine which type of HB we are dealing with-it may be a codimension 12 bifurcation-at $f_{1} \approx 2.57 \times 10^{-5}$, indirect evidence, such as the existence of hysteresis, and thereby irreversibility in terms of $f_{1}$, suggests that it is subcritical. (If we start on the $\mathbf{x}_{\mathrm{DS}}^{*}$-curve and increase $f_{1}$ beyond the fold bifurcation value of $f_{1}$, we will witness a (hysteresis) jump to $\mathbf{x}_{H}^{*}$. However, if we start on $\mathbf{x}_{H}^{*}$ at an $f_{1}$ value greater than the fold bifurcation value and reduce the value of $f_{1}$ to less than the fold bifurcation value, we will not see a similar jump, not even as $f_{1}$ is decreased below the HB value.)

In many systems hysteresis is not a desirable feature, but in this case its presence is more than welcome, because the irreversibility that it entails implies that if we can increase $f_{1}$ beyond the HB point, the system will tend to the healthy state, $\mathbf{x}_{H}^{*}$. Put in terms of the NOD mouse: without prophylactic measures, the $\beta$ cells of a NOD mouse are destined to be depleted by a chronic inflammation (within the frame of the DuCa model). However, by artificially increasing the phagocytic ability of its resting macrophages after the inflammation has become chronic, even just for a while, the reservoir of apoptotic $\beta$ cells would be cleared, thereby bringing the inflammation to an end. See Section 8 for a discussion of the effectiveness, and realism, of this method.

While bifurcation diagrams for all variables have been compiled, the only other diagram we choose to present in Fig. 2 features apoptotic $\beta$ cells. This is due to their key role - they are in a sense the heart of the model.

Figure 2 provides an idea of how many apoptotic $\beta$ cells need to be present at a given time to induce chronic inflammation in the NOD mouse. In other words, it provides information about how little the apoptotic wave could be, while still driving the system toward $\mathbf{x}_{\mathrm{DS}}^{*}$. So for a given phagocytosis rate we can use it to calculate the amount of apoptotic $\beta$ cells needed to push the system into the basin of attraction of $\mathbf{x}_{\mathrm{DS}}^{*}$.

To make things more precise, let $f_{1, \mathrm{hb}}$ be the HB value of $f_{1}$ and let us denote the line of low-level inflammation $B_{a}$-LI. Then we can define the domain in which solutions are attracted to $\mathbf{x}_{\mathbf{H}}^{*}$ as

$$
W=\left\{\left(f_{1}, B_{a}\right) \mid 0<f_{1}<f_{1, \mathrm{hb}} \wedge B_{a}>B_{a}-\mathrm{LI}\right\} .
$$

In Fig. 2 this set is outlined by the shaded area. This set will prove important later. Next we present, and comment on, a bifurcation diagram where the phagocytosis rate of activated macrophages is used as the bifurcation parameter.

Using $f_{2}$ as the bifurcation parameter: First of all we remark that $f_{2}$-the phagocytosis rate of activated macrophages - is estimated to be within the bistable region $\left(f_{2}=1 \times 10^{-5}\right.$ for NOD mice), so it agrees with the fact that a chronic inflammation for NOD mice is reached, when the apoptotic wave stimulates the system to exceed the threshold of unstable fixed points. Again we have taken the natural logarithm of the $M_{a}$ concentration to get a clearer view of the distinction between the branch of low-level inflammation and the healthy, or inflammation-free, state, which is stable for all values of $f_{2}$. We notice some (qualitative) similarities as well as differences between Fig. 3 and its $f_{1}$-counterpart 


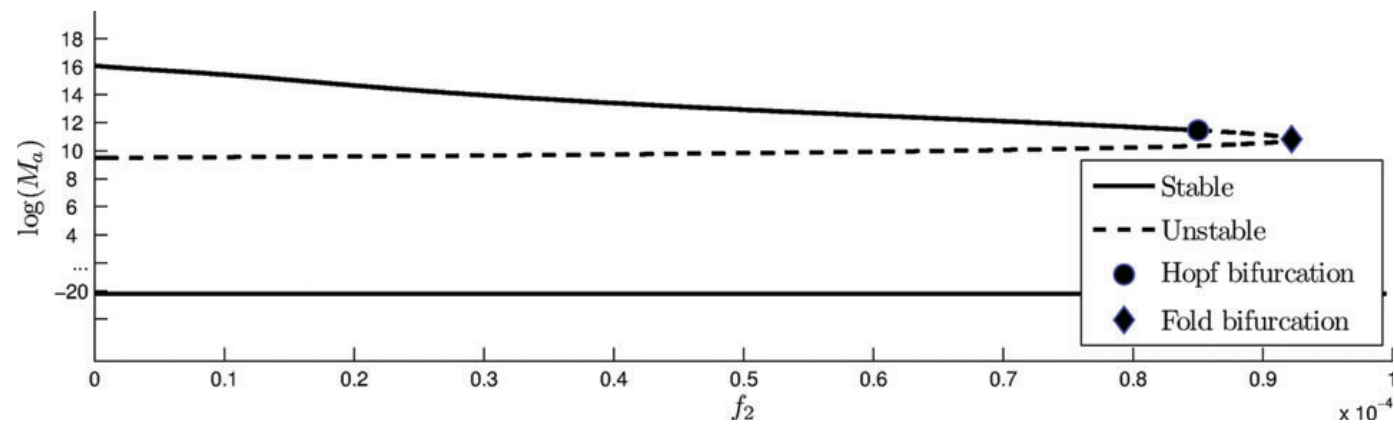

FIG. 3. Bifurcation diagram showing $\log _{e}\left(M_{a}\right)$ versus the phagocytosis rate of activated macrophages, $f_{2}$. All other parameters are as given in Table A1. Notice that the y-axis has been modified to facilitate the overview. The three dots on the y-axis indicate the jump in range. Solid lines consist of stable fixed points. Dashed lines indicate unstable fixed points. The uppermost line is the disease state, and the lowest line is the disease-free state. They are separated by a state of low-level inflammation, which is unstable. Two bifurcations occur as $f_{2}$ is varied. At $f_{2} \approx 8.48 \times 10^{-5}$ a HB occurs (indicated by filled circle), and a fold bifurcation happens at $f_{2} \approx 9.22 \times 10^{-5}$ (indicated by filled diamond). The system is bistable for $f_{2}$ values lower than the Hopf point.

in Fig. 2. Let us start with the resemblances: the system has two stable states for $f_{2}<8.48 \times 10^{-5}$ a disease-free state and a disease state. Between the two is a threshold of unstable states, which, as in Fig. 2, is interpretable as a state of low-level inflammation. At $f_{2} \approx 8.48 \times 10^{-5}$ the disease state undergoes a $\mathrm{HB}$, making it unstable.

Eventually the two unstable branches annihilate each other in a fold bifurcation, at $f_{2} \approx 9.25 \times 10^{-5}$, after which only the healthy rest state remains. Finally, both figures exhibit hysteresis.

A clear difference, between using the phagocytosis rate of activated macrophages as bifurcation parameter and using the phagocytosis rate of resting macrophages, is the magnitude by which we have to increase the bifurcation parameter to reach a HB along the disease state; $f_{1, \mathrm{hb}} \approx 2.57 \times 10^{-5}$ compared with $f_{2, \mathrm{hb}} \approx 8.48 \times 10^{-5}$. To put this into perspective, the average phagocytosis rate of activated Balb/c macrophages is $5 \times 10^{-5}$ (Marée et al., 2006).

To gain insight into the dynamics near the HB point, we estimated $f_{2, \text { hb }}$, and the values of the variables close to the HB using XPPAUT. Based on these values we ran simulations in MATLAB for different initial values of $M$ and $M_{a}$ to see how such perturbations behave. Figures 4 and 5 show two trajectories in the $M-M_{a}$ phase plane and $M(t)$, respectively, for different perturbations (denoted P1 and P2), in the $M$ and $M_{a}$ variables, away from $\mathrm{x}_{\mathrm{DS}}^{*}$, with $f_{2}=8.477652 \times 10^{-5}$-this is the value XPPAUT gives as the $\mathrm{HB}$ value of $f_{2}$. Observe that the curve starting at $\mathrm{P} 1$ is attracted to the disease state, while the trajectory from $\mathrm{P} 2$ is attracted to a limit cycle. This is also evident from Fig. 5. The solution curve from $\mathrm{P} 1$ exhibits transiently growing oscillations that eventually dampen and tend to $\mathbf{x}_{\mathrm{DS}}^{*}$. The P2-solution, on the other hand, grows to sustained oscillations with an amplitude of $\approx 5420$ resting macrophages and a period of $\approx 18$ days; the limit cycle can be interpreted as a disease state with some days seeing a higher level of inflammation than others. The fate of the two perturbations shows that phase space is separated into sets with different long-term behaviors. An attempt at estimating the boundaries of the different sets, through numerical investigation, revealed that the situation is quite complex. Within the set of perturbations that tend to the stable limit cycle small islands exist, which consist of points that tend to $\mathbf{x}_{\mathrm{DS}}^{*}$. In Appendix B we provide a figure of a larger part of the $M-M_{a}$ phase plane, a plot of how perturbations in $B_{a}, B_{n}$ and $C$ behave, as well as a brief discussion of the dynamics near $f_{2, \mathrm{hb}}-\mathrm{a}$ full bifurcation analysis is beyond the scope of this paper. 


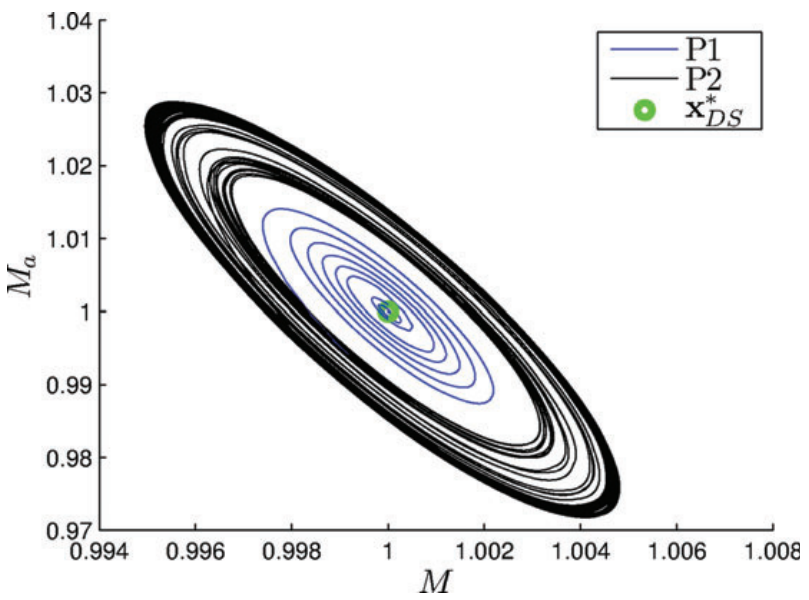

FIG. 4. A zoom of the $M-M_{a}$ phase plane for $f_{2}=8.477652 \times 10^{-5}$. P1 and P2 denote perturbations, in the $M$ and $M_{a}$ directions, from $\mathbf{x}_{\mathrm{DS}}^{*}$. Curves have been scaled relative to $\mathbf{x}_{\mathrm{DS}}^{*}$.

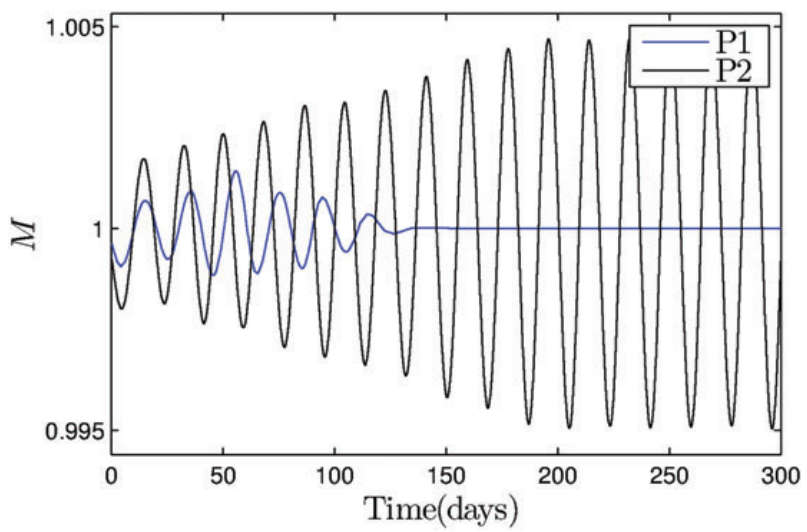

FIG. 5. Plot of $M(t)$ for the initial conditions P1 and P2. The 'P1-solution' settles at $\mathbf{x}_{\mathrm{DS}}^{*}$, while the P2-solution approaches a stable limit cycle. Curves have been scaled relative to $\mathbf{x}_{\mathrm{DS}}^{*}$.

Next we analyse how the bifurcation points behave as we change both phagocytosis rates, while keeping all other parameters fixed. The impetus for doing so comes from the therapeutic perspective: assume that we are able to manipulate the phagocytosis rates in NOD mice. Then, even if this were the case, Figs 2 and 3 show that if we were to abrogate inflammation via this method, we need to increase the individual phagocytosis rates in excess of the rates observed in Balb/c mice. Thus, the question, from a medical point of view, becomes: is there a golden mean that is physiologically reasonable?

\section{Varying $f_{1}$ and $f_{2}$ simultaneously: codimension 2 analysis}

As stated we may conceive of not being able to induce great enough changes in the phagocytosis rates by themselves to arrest the inflammation. The natural cause of action is thus to investigate what happens 


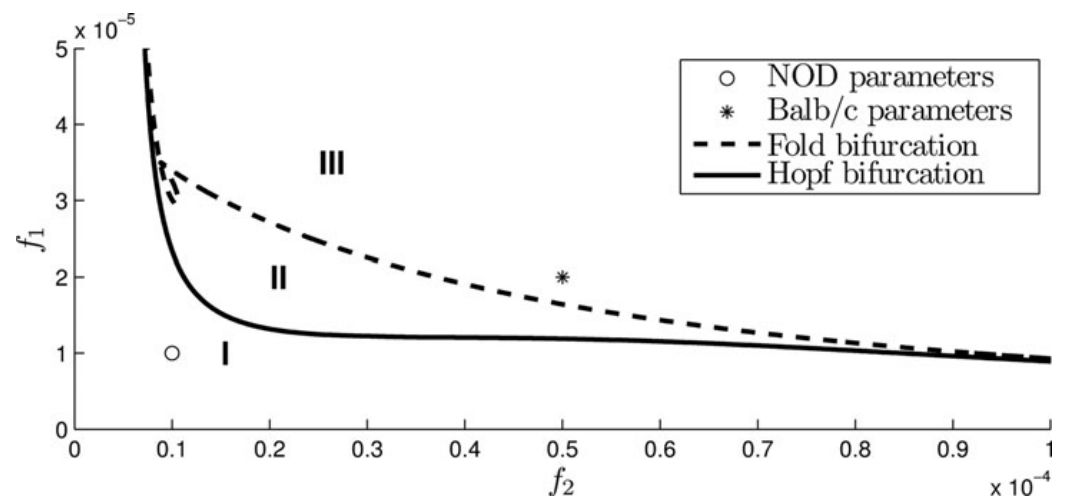

FIG. 6. Codimension 2 diagram with the phagocytosis rate of resting macrophages, $f_{1}$, plotted against the phagocytosis rate of activated macrophages, $f_{2}$. Along the solid line are HBs, while fold bifurcations exist along the dashed line. The $\left(f_{1}, f_{2}\right)$ combinations of NOD and Balb/c mice are indicated by a circle and an asterisk, respectively. I indicates the bistable domain, II has two unstable states and one stable - the healthy state — and in III only the healthy state exists.

as the rates are varied simultaneously. Due to the complexity of the system, an analytic codimension 2 analysis is impossible. Therefore a numerical codimension 2 analysis must suffice.

Figure 6 was produced using XPPAUT and MATLAB. Crossing the solid line entails a HB, while the dashed line consists of fold bifurcations. This means that for combinations of $\left(f_{1}, f_{2}\right)$ in the domain denoted by I in Fig. 6 the system has two stable branches and one unstable, if $\left(f_{1}, f_{2}\right)$ belong to domain II the system has two unstable branches and one stable, while $\left(f_{1}, f_{2}\right)$-combinations in III leaves the system with only one branch of stability, namely $\mathbf{x}_{H}^{*}$. In Fig. 6 we have added a circle that indicates the $\left(f_{1}, f_{2}\right)$ values of NOD mice, and an asterisk that shows the phagocytosis rates of Balb/c macrophages. We see that NOD mice lie in domain I, while Balb/c mice are in domain III. Interestingly, the Balb/c mice would still not develop a chronic inflammation even without an increase in phagocytic capability following activation; i.e. no inflammation would develop with the parameter combination $\left(f_{1}, f_{2}\right)=$ $\left(2 \times 10^{-5}, 2 \times 10^{-5}\right)$.

Though the line of fold bifurcations is interesting from a mathematical point of view due to the apparent cusp catastrophe, the same is not the case when we look at it from a medical perspective. This is because in order to cross the line of fold bifurcations by increasing $f_{1}$ or $f_{2}$ (or both) we would be moving along an unstable branch, once we enter domain II. So while this is possible in theory, it becomes impossible in practice since the actual in vivo system will be perturbed constantly, thus we would not be able to stay on the unstable branch. The underlying simulations of Fig. 6 were made with the initial condition $\mathbf{x}(0)=\left(4.77 \times 10^{5}, 0,0,0,0\right)^{\mathrm{T}}$, which is the (physiological) initial condition given by Marée et al. (2006).

By defining

$$
\mathrm{I}_{s}=\left\{\left(f_{1}, f_{2}\right) \mid \mathbf{x}(\mathbf{f}, t) \rightarrow \mathbf{x}_{\mathrm{DS}}^{*} \text { for } t \rightarrow \infty\right\},
$$

i.e. $\mathrm{I}_{s}$ is the subset of $\mathrm{I}$, where $\mathbf{x}_{\mathrm{DS}}^{*}$ is attracting, and

$$
\mathrm{II}=\left\{\left(f_{1}, f_{2}\right) \mid \mathbf{x}(\mathbf{f}, t) \rightarrow \mathbf{x}_{H}^{*} \text { for } t \rightarrow \infty\right\},
$$

where $\mathbf{f}=\left(f_{1}, f_{2}\right)$, we are able to give a description of the line of HBs in terms of these two domains. Since the HB line, $\left(f_{1, \mathrm{hb}}, f_{2, \mathrm{hb}}\right)$, is not a part of $\mathrm{I}_{s}$ nor II and, furthermore, the axes are not a part of $\mathrm{I}_{s}$, we 


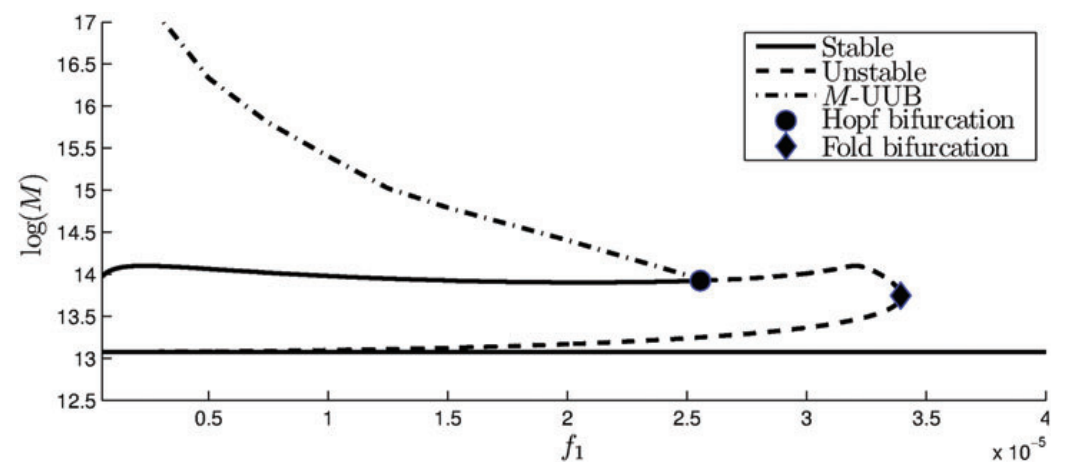

FIG. 7. Bifurcation diagram with the logarithm of $M$ versus $f_{1}$ - the line with estimated turning concentrations, denoted M-UUB, has been added. We have taken the natural logarithm of the concentrations to facilitate the overview. The lower unstable branch does not merge with the healthy state as would appear.

see that $\mathrm{I}_{s}$ and II are open sets. Thus, we can characterize the HB curve as follows

$$
\mathfrak{L}=\partial \mathrm{I}_{s} \cap \partial \mathrm{II},
$$

where $\partial$ denotes the boundary of the sets. Also notice that by these definitions we have

$$
\mathrm{I}_{s} \cap \mathrm{II}=\emptyset .
$$

\section{An important feature of the system}

Let $M_{a}^{\text {DS }}$ denote the stable disease branch in Fig. 2 and $f_{1, \text { hb }}$ be the value of $f_{1}$ at the HB point. Then for values of $M_{a}$ slightly above $M_{a}^{\mathrm{DS}}$ to the left of, but close to, $f_{1, \mathrm{hb}}$, it turns out that $\mathbf{x}(t) \rightarrow \mathbf{x}_{H}$ as $t \rightarrow \infty$. In other words a perturbation that increases $M_{a}$ may push $\mathbf{x}(t)$ into a domain where the system tends towards the healthy state, $\mathbf{x}_{H}^{*}$. In fact there is a demarcation curve $M_{a}^{\mathrm{DS}}\left(f_{1}\right)+K_{a}\left(f_{1}\right)$, with $K_{a}\left(f_{1}\right)>0$ and $K_{a}\left(f_{1}\right) \rightarrow 0$ for $f_{1} \rightarrow f_{1, \mathrm{hb}}$ from the left, such that whenever $M_{a}\left(f_{1}\right)>M_{a}^{\mathrm{DS}}\left(f_{1}\right)+K_{a}\left(f_{1}\right)$ for $0<f_{1}<f_{1, \mathrm{hb}}$, then $\mathbf{x}(t) \rightarrow \mathbf{x}_{H}^{*}$ as $t \rightarrow \infty$.

Put in laymen terms: very near the HB point $\mathbf{x}(t)$ tends to $\mathbf{x}_{H}^{*}$ as $t$ tends to infinity, provided that $M_{a}(0)$ is perturbed to be slightly above $M_{a}^{\mathrm{DS}}$. At lower $f_{1}$-values we need to use a higher $M_{a^{-}}$ concentration to induce the same behaviour. The theme repeats itself when analysing the bifurcation diagram that depicts $M$ versus $f_{1}$. For future reference we will call the line of 'turning concentrations' the upper unstable branch (UUB).

In Fig. 7 we have added the $M$-UUB to a bifurcation diagram of $M$ versus $f_{1}$. Notice that we have, again, taken the natural logarithm of the concentration to ease the overview. It should be pointed out that the line of low-level inflammation does not merge with the healthy state - it is a result of weighing the $M$-UUB over details along the healthy state. Based on extensive numerical investigations, we state the following conjecture without proof.

Conjecture 7.1 Let $x_{i} \geqslant 0, i=M_{a}, B_{n}, C$, if $B_{a}(0) \in W$ and $M(0) \in \mathbb{R}^{+} \backslash\left(0 ; M\right.$-UUB), where $B_{a}(0)$ is the initial value of $B_{a}$. Then $\mathbf{x}(t) \rightarrow \mathbf{x}_{H}^{*}$ for $t \rightarrow \infty$.

The reason why $M(0) \in \mathbb{R}^{+} \backslash(0 ; M$-UUB) instead of having $M(0)$ in the range between the lowinflammatory state and the $M$-UUB is that if $M(0)$ is in the range between the healthy state and the 
low-level inflammatory state while $B_{a}(0) \in W$, then at some time $t^{\prime}, M\left(t^{\prime}\right)$ will have traversed low-level inflammation and as $t \rightarrow \infty$ we have $M \rightarrow M^{\text {DS }}$.

By the same token, if $B_{a} \in W$ and $M \in\left[0 ; M\right.$-UUB), then $\mathbf{x}(t) \rightarrow \mathbf{x}_{\mathrm{DS}}^{*}$ for $t \rightarrow \infty$. Thus, no matter how many apoptotic $\beta$ cells we choose to put into the system, we still need only to exceed the $M$-UUB to reverse the inflammation, which is quite remarkable. A similar statement holds for the $M_{a}$-UUB.

\section{Biological interpretation: Therapeutic prospects}

Modifying $f_{1}$ and/or $f_{2}$ : The codimension 1 bifurcation diagrams illustrate what we had anticipated. Namely that increasing the phagocytosis rates sufficiently entails a shift in long-term behavior: for $f_{1}<$ $f_{1, \mathrm{hb}} \wedge f_{2}=1 \times 10^{-5}$ (or $f_{2}<f_{2, \mathrm{hb}} \wedge f_{1}=1 \times 10^{-5}$ ), the system tends to a fixed point that corresponds to chronic inflammation when physiological initial conditions are used, while for $f_{1}>f_{1, \mathrm{hb}}\left(f_{2}>f_{2, \mathrm{hb}}\right)$, the system tends to a fixed point that corresponds to a healthy individual regardless of the choice of initial conditions.

From a medical standpoint the most important facet of Figs 2 and 3 is that they allow us establish which of the phagocytosis rates it is most opportune to manipulate to stop an inflammation. Comparing Fig. 2 with 3, we find that, if we can only adjust one of the phagocytosis rates in the NOD model, enhancing $f_{1}$ seems most opportune. By this we mean that $f_{1}$ needs 'only' to be changed to $\sim 2.57 \times 10^{-5}$ to arrest and reverse any inflammation, whereas $f_{2}$ must exceed a staggering $8.45 \times 10^{-5}$ to induce the same effect when $f_{1}$ is kept at $1 \times 10^{-5}$. Furthermore, due to the irreversibility of the system, we would only need to enhance the phagocytic ability of the resting macrophages until they have phagocytized enough apoptotic $\beta$ cells, as to make the concentration of activated macrophages drop beneath the lower branch of unstable fixed points; cf. Fig. 2. Here we should remark that when the macrophagedriven inflammation has become chronic, the pool of healthy resting $\beta$ cells is still sufficient to maintain normoglycaemia. This implies that arresting inflammation at this point in time should mean that hyperglycaemia does not develop - provided that clearance of apoptotic $\beta$ cells happens sufficiently fast; cf. Fig. 8. To establish how fast the chronic inflammation recedes upon altering $f_{1}$, we did a simulation with $f_{1}=2.56 \times 10^{-5}<f_{1, \mathrm{hb}}$, and ran it until the 5 concentrations had stabilized at values that correspond to chronic inflammation. We took these values and used them as initial values for a simulation where $f_{1}=2.7 \times 10^{-5}$. Figuratively speaking, we froze the mouse in time, manipulated the phagocytic ability of its resting macrophages and then unfroze it, or put another way: we bumped up the phagocytosis rate instantaneously after inflammation had settled in.

The simulation of the time right after the phagocytosis rate has been increased is seen in Fig. 8 . It is noticeable that the inflammation is immediately reversed, and a healthy state is reached when approximately 6 days have transpired-there are still activated macrophages present, but they only linger for as long as it takes them to be deactivated.

Adding macrophages: The finding that adding enough (resting or activated) macrophages to the system, after it has settled at $\mathbf{x}_{\mathrm{DS}}^{*}$, can act as a perturbation that drives the system into a domain where $\mathbf{x}_{H}^{*}$ is attracting was an unexpected consequence of the mathematical analysis. The notion of medical researchers was that adding macrophages should exacerbate inflammation-not terminate it. So our result appeared counter-intuitive at first. Nevertheless, adding macrophages seems to be a straightforward and effective cure, as is demonstrated in Fig. 9, where we see how a one-time administration of a bolus of resting macrophages causes the inflammation to die out within 20 days. To produce Fig. 9 , we ran a simulation with $f_{1}=2.5 \times 10^{-5}$ and physiological initial conditions until the system had settled at $\mathbf{x}_{\mathrm{DS}}^{*}$. Then we started a new simulation with $\mathbf{x}(0)=\mathbf{x}_{\mathrm{DS}}^{*}$ except we increased the initial condition for resting macrophages from $1.109330 \times 10^{6}$ to $1.187451 \times 10^{6}$, i.e. an increase of 78120 macrophages. 


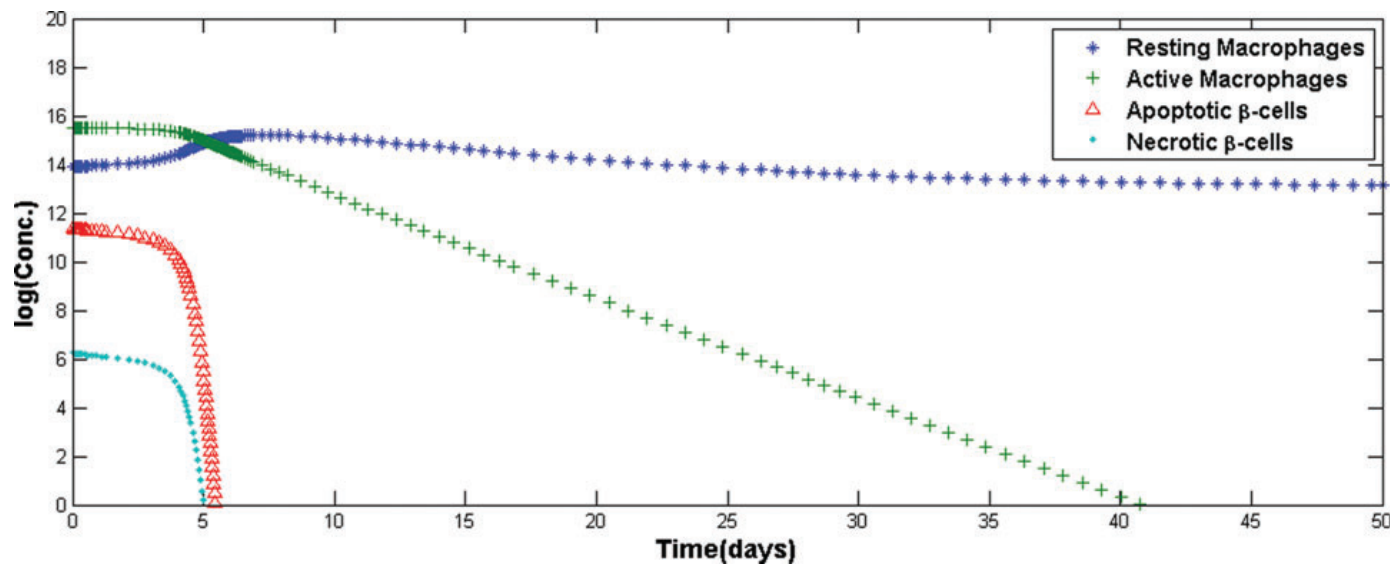

FIG. 8. A simulation showing the effectiveness of raising the phagocytic capability of resting macrophages in NOD mice from $f_{1}=1 \times 10^{-5}$ to $f_{1}=2.7 \times 10^{-5}$ at a time where chronic inflammation has otherwise been established. Notice the extinction of apoptotic and necrotic $\beta$ cells in 5-6 days. Cytokines are not visible on the figure because their concentration is too low.

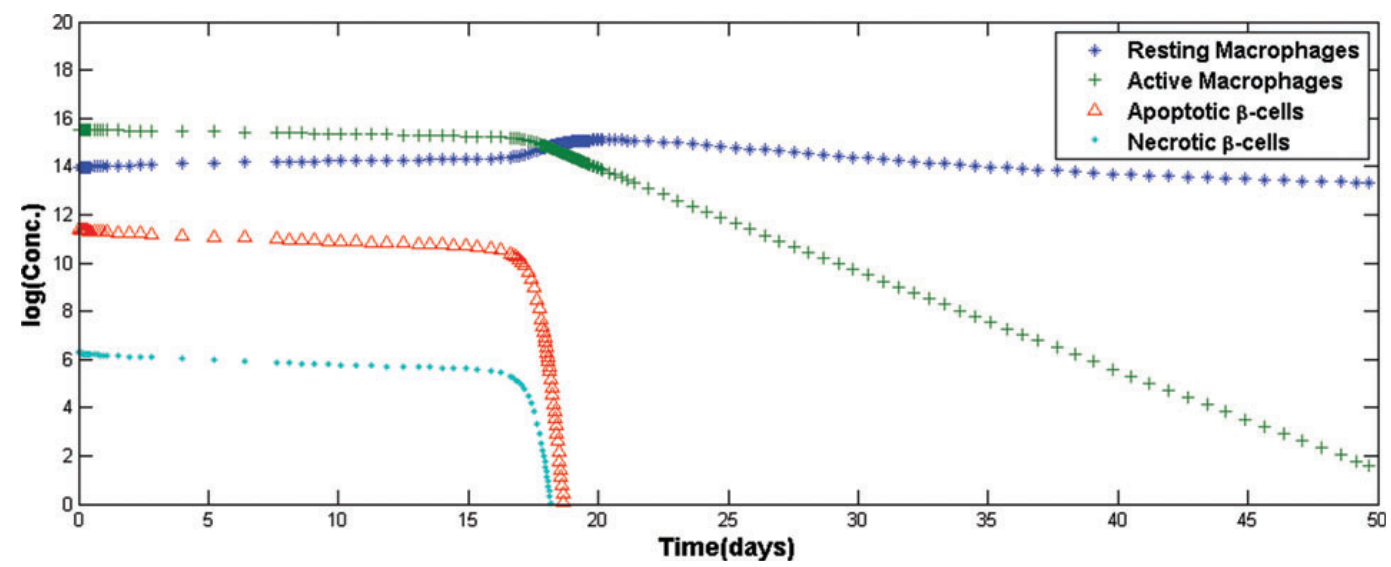

FIG. 9. Simulation showing how many days it takes an otherwise chronic inflammation to abate and disappear upon addition of resting macrophages to a site of inflammation. Notice the extinction of apoptotic and necrotic $\beta$ cells within 20 days. Cytokines are not visible on the figure because their concentration is too low.

We see that it takes about 3 times longer for the inflammation to subside compared with that which we saw when we simply increased the phagocytosis rate.

\section{Discussion}

The basis for this article is the work of Marée et al. (2006), who proposed a mathematical model of type 1 diabetes. Based on data, obtained from a mouse strain that develops T1D (NOD) and one that does not $(\mathrm{Balb} / \mathrm{c})$, they were able to demonstrate that the difference between developing T1D or not is related to defective macrophages (Marée et al., 2006). 
We have elaborated on their work, mathematically,

- by demonstrating that the DuCa model satisfies positivity, in the sense $x_{i}(t) \geqslant 0$ for all $t>0$ and $i=1, \ldots, 5$, if $x_{i}(0) \geqslant 0$ for all $i=1, \ldots, 5$ and

- by constructing a family of attracting trapping regions in $\left(\mathbb{R}_{+} \cup\{0\}\right)^{5}$.

Taken together, these results are tantamount to (weak) persistence and adds to the trustworthiness of the DuCa model. This analysis in conjunction with our bifurcation analysis, which provides important medical insights, as discussed below, accounts for the novelty of our work.

We based our bifurcation analysis on the phagocytosis rates $f_{1}$ and $f_{2}$, since these two parameters constitute the only difference between NOD mice and Balb/c mice that do not suffer from T1D during their lifetime. In accordance with what one would expect, we found that by increasing the phagocytosis rates either by themselves or simultaneously, inflammation in NOD mice could be reversed. These results are obviously not surprising. However, we have also addressed the question of whether it is at all possible to increase the phagocytosis rates.

One hypothetical method is to introduce activated Balb/c-macrophages into the islets of Langerhans of an NOD mouse suffering from chronic inflammation of the $\beta$ cells. This way the average phagocytosis rate could exceed $f_{1, \mathrm{hb}}$. However, such allogenic transfer has the problem of immunoincompatibility, and we point instead to the result that we find to be the most interesting. Namely, transfer of syngeneic macrophages, which, despite their lower phagocytic potential, could be used. These could be harvested from non-diabetic NOD mice (resting macrophages) or from pre-diabetic or diabetic mice (activated macrophages). The technical feasibility of this is of course not trivial though isolation and injection of macrophages should be straightforward. The major hurdle would be the method of administration, and while it is beyond the scope of this article to delve into this problem, we propose injecting them in the retroperitoneal space or administering them intravenously; we speculate that a substantial part of injected macrophages will home to the inflamed pancreatic islets of the recipient. Injecting the macrophages directly into the pancreas seems inviable due to the delicateness of the organ.

A natural objection that critics may hold to our strategy is that the method outlined could suffer the drawback that there simply is not room enough for the amount of additional macrophages needed. To this end, we refer to Poulter \& Turk (1975), who give an estimate of the volume of a macrophage to be $1450 \mu \mathrm{m}^{3}$ (Poulter \& Turk, 1975). This converts into $1.45 \times 10^{-9} \mathrm{ml}$. Hence, based on the result of Poulter \& Turk (1975), there is room for 689655172 macrophages within $1 \mathrm{ml}$-at $f_{1}=2.5 \times 10^{-5}$ we added 80000 macrophages, or approximately $0.1 \mu \mathrm{L}$, to bring the inflammation to an end. Thus, the volume of supplementary macrophages does not speak against this approach as a viable means of inducing health in NOD mice with chronic islet inflammation; especially if it can be combined with some way of enhancing the phagocytic ability of the macrophages.

In some of the mathematical excursions above we speak about $t$ going to infinity, which may lead some readers to wonder whether our results have any merit beyond the mathematical realm. In other words, we need to consider the biology of the model, and by extension, our treatment strategies are intended to imitate. That is, it may be that our treatments work mathematically, but if the healthy state is not reached at least within the lifespan of the average laboratory mouse, then the methods are, all in all, useless. The median lifespan of a mouse is approximately 30 months (Blüher et al., 2003), and we see that following either of our hypothetical intervention methods, the healthy state is reached within days to a few weeks of treatment. So our approaches live up to this criterion.

However, a stricter, and more appropriate, criterion is that the inflammation should be able to subside before T1D becomes overt, and while there are still enough healthy $\beta$ cells left to maintain 
normoglycemia. Hyperglycemia occurs when the functional $\beta$ cell mass has been reduced to $10-40 \%$ of its original size (Seeley et al., 2008). This happens at around 30 weeks of age in NOD mice (Sreenan et al., 1999). If our method of adding macrophages, or increasing the phagocytosis rate(s), is applied in due time, i.e. on the path to or shortly after chronic inflammation is established, inflammation will be cleared within 20 days, as evidenced by Figs 8 and 9. Importantly at this very early stage of disease, the population of healthy $\beta$ cells remains relatively stable (Rudy et al., 1997), probably due to increased proliferation. (Rat $\beta$ cells exhibit an increase in proliferation under mild hyperglycemia Hügl et al., 1998.) Thus, sufficiently many $\beta$ cells will remain to keep serum glucose levels below hyperglycemic levels. After this no further therapeutic steps should be needed. The high plasticity of $\beta$ cells also suggests that it may be possible that following resolution of inflammation, mild hyperglycemia will stimulate the remaining $\beta$ cells to proliferate, and possibly replenish some of the lost cells. This also points to a possible, natural, extension of the model; namely adding an equation describing the healthy $\beta$ cells. However, as described above, this population remains at a quasi steady state during the initial period of macrophage-induced $\beta$ cell apoptosis (and also during initial $\mathrm{T}$ cell infiltration). So adding the equation to the DuCa model should not change the long-term behaviour. But it would be an interesting addition in a more elaborate model. ( $\beta$ cells have been modelled explicitly in other models; see, e.g., Wang et al. (2006) and Mahaffy \& Edelstein-Keshet (2007) for different approaches to modelling healthy $\beta$ cells in T1D.)

In the greater scope of things, i.e. applying these ideas to humans, it is paramount that these prophylactic measures are employed prior to the onset of frank diabetes, which occurs when approximately $60-90 \%$ of the $\beta$ cell population has been destroyed. Put another way: identifying pre-diabetic humans is key. Fortunately, doing so is no longer science fiction as evidenced in the article by Knip et al. (2010). Furthermore, just like rodents, humans can also maintain blood glucose homeostasis even when a substantial portion of the functional $\beta$ cells have been depleted, meaning that the time window of utilizing our ideas is not confined to, e.g., a few weeks, but rather months or perhaps, even, years. We expect that as our understanding of T1D and its precursors evolves, it will become easier and safer to pinpoint the opportune moment, or period, for administration of syngeneic macrophages.

In this article we have presented, basically, two different methods of interfering with the progression to T1D. Both approaches have been analysed in a setting prior to infiltration by $\mathrm{T}$ cells- the main mediators of $\beta$ cell death-which occurs some time after the establishment of persistent $\beta$ cell death driven by macrophage-derived proinflammatory cytokines. However, even after T cell-mediated $\beta$ cell destruction has been initiated, we still propose introducing additional macrophages, since the overall increase in phagocytosis will reduce the feedback from apoptotic and necrotic $\beta$ cells to priming and activation of T cells, possibly shutting off this feedback all together.

In conclusion, while we recognize that our strategies may be some years from human trials, results published since our first submission demonstrate that at least one of our proposed methods has merit to it, namely the method of supplying macrophages (Parsa et al., 2012). In addition to corroborating our results, and confirming that mathematical modelling and analysis can, in fact, provide significant insights into diseases, it leaves us optimistic for the perspective of developing a cure that may benefit the millions of people who, in the future, would otherwise have developed T1D.

\section{REFERENCES}

Alberts, B., Johnson, A., Lewis, J., Raff, M., Roberts, K. \& Walter, P. (2008) Molecular Biology of The Cell, 5th edn. New York: Garland Science. 
BlÜher, M., Kahn, B. \& Kahn, C. (2003) Extended longevity in mice lacking the insulin receptor in adipose tissue. Science, 299, 572.

Bock, T., Kyhnel, A., Pakkenberg, B. \& Buschard, K. (2003) The postnatal growth of the beta-cell mass in pigs. J. Endocrinol., 179, 245.

Clewley, R., Sherwood, W., LaMar, M. \& Guckenheimer, J. (2007) Pydstool, a software environment for dynamical systems modeling. http://pydstool.sourceforge.net (last accessed April 15, 2013).

De Boer, R. \& Perelson, A. (1994) T cell repertoires and competitive exclusion. J. Theoret. Biol., 169, 375-390.

Ermentrout, B. (1987) Simulating, Analyzing, and Animating Dynamical Systems: A Guide to XPPAUT for Researchers and Students, Vol. 14. Philadelphia, PA: Society for Industrial Mathematics.

Freiesleben De Blasio, B., BaK, P., Pociot, F., Karlsen, A. \& Nerup, J. (1999) Onset of type 1 diabetes: a dynamical instability. Diabetes, 48, 1677.

Höglund, P., Mintern, J., Waltzinger, C., Heath, W., Benoist, C. \& Mathis, D. (1999) Initiation of autoimmune diabetes by developmentally regulated presentation of islet cell antigens in the pancreatic lymph nodes. J. Exp. Med., 189, 331-339.

HÜGL, S., White, M. \& RHODES, C. (1998) Insulin-like growth factor i (igf-i)-stimulated pancreatic $\beta$-cell. The $J$. Biol. Chem., 273, 17771-17779.

Kassem, S., Ariel, I., Thornton, P., Scheimberg, I. \& Glaser, B. (2000) Beta-cell proliferation and apoptosis in the developing normal human pancreas and in hyperinsulinism of infancy. Diabetes, 49, 1325.

Knip, M., Korhonen, S., Kulmala, P., Veijola, R., Reunanen, A., Raitakari, O., Vitikari, J. \& Åkerblom, H. (2010) Prediction of type 1 diabetes in the general population. Diabetes Care, 33, 1206.

Lo, J. \& Clare-Salzler, M. J. (2006) Dendritic cell subsets and type i diabetes: Focus upon dc-based therapy. Autoimmun. Rev., 5.

Mahaffy, J. \& Edelstein-Keshet, L. (2007) Modeling cyclic waves of circulating T cells in autoimmune diabetes. SIAM J. Appl. Math., 67, 915-937.

Maier, L. \& Wicker, L. (2005) Genetic susceptibility to type 1 diabetes. Curr. Opin. Immunol., 17, 601-608.

Marée, A. F., Komba, M., Dyck, C., Labecki, M., Finegood, D. T. \& Edelstein-Keshet, L. (2005) Quantifying macrophage defects in type 1 diabetes. J. Theoret. Biol., 233, 533-551.

Marée, A. F. M., Komba, M., Finegood, D. T. \& Edelstein-Keshet, L. (2008) A quantitative comparison of rates of phagocytosis and digestion of apoptotic cells by macrophages from normal (balb/c) and diabetesprone (nod) mice. J. Appl. Physiol., 104, 157-169.

Marée, A. F. M., Kublik, R., Finegood, D. T. \& Edelstein-Keshet, L. (2006) Modelling the onset of type 1 diabetes: can impaired macrophage phagocytosis make the difference between health and disease? Phil. Trans. R. Soc., 364, 1267-1282.

Mathis, D., Vence, L. \& Benoist, C. 2001. Beta-cell death during progression to diabetes. Nature, 414, 792-798.

Parsa, R., Andresen, P., Gillett, A., Mia, S., Zhang, X., Mayans, S., Holmberg, D. \& Harris, R. (2012) Adoptive transfer of immunomodulatory $\mathrm{m} 2$ macrophages prevents type 1 diabetes in nod mice. Diabetes, $\mathbf{6 1}$, 2881-2892.

Poulter, L. \& TuRK, J. (1975) Rapid quantitation of changes in macrophage volume induced by lymphokine in vitro. Clin. Exp. Immunol., 19, 193.

Rudy, G., Sutherland, R. \& Lew, A. (1997) Temporal discontinuities in progression of nod autoimmune diabetes. Immunol. Res., 16, 137-147.

Scaglia, L., Cahill, C., Finegood, D. \& Bonner-Weir, S. (1997) Apoptosis participates in the remodeling of the endocrine pancreas in the neonatal rat. Endocrinology, 138, 1736.

Seeley, R. R., Stephens, T. D. \& Tate, P. (2008) Anatomy \& Physiology, 8th edn. New York: McGraw-Hill.

SKYLER, J. (2007) Prediction and prevention of type 1 diabetes: progress, problems, and prospects. Clin. Pharmacol. Therapeut., 81, 768-771.

Sparre, T., Larsen, M., Heding, P., Karlsen, A., Jensen, O. \& Pociot, F. (2005) Unraveling the pathogenesis of type 1 diabetes with proteomics: present and future directions. Mol. Cell. Proteom., 4, 441.

Sreenan, S., Pick, A., Levisetti, M., Baldwin, A., Pugh, W. \& Polonsky, K. (1999) Increased beta-cell proliferation and reduced mass before diabetes onset in the nonobese diabetic mouse. Diabetes, 48, 989. 
Steer, S. A., Scarim, A. L., Chambers, K. T. \& Corbett, J. A. (2006) Interleukin-1 stimulates $\beta$-cell necrosis and release of the immunological adjuvant hmgb1. PLoS Med., 3, 253-266.

Stoffels, K., Overbergh, L., Giulietti, A., Kasran, A., Boulllon, R., Gysemans, C. \& Mathieu, C. (2004) Nod macrophages produce high levels of inflammatory cytokines upon encounter of apoptotic or necrotic cells. J. Autoimmun., 23, 9-15.

Trudeau, J. D., Dutz, J. P., Arany, E., Hill, D. J., Fieldus, W. E. \& Finegood, D. T. (2000) Neonatal beta-cell apoptosis a trigger for autoimmune diabetes? Diabetes, 49, 1-7.

WANG, X., He, Z. \& GHosh, S. (2006) Investigation of the age-at-onset heterogeneity in type 1 diabetes through mathematical modeling. Math. Biosci., 203, 79-99.

Wild, S., Roglic, G., Green, A., Sicree, R. \& King, H. (2004) Global prevalence of diabetes-estimates for the year 2000 and projections for 2030. Diabetes Care, 27, 1047-1053.

Zong, W. \& Thompson, C. (2006) Necrotic death as a cell fate. Genes Dev., 20, 1.

\section{Appendix A. Model parameters}

The parameters, their meaning and their values are given in Table A1.

TABLE A1 Model-parameters ${ }^{\dagger}$, values and units for NOD mice

\begin{tabular}{llcc}
\hline Parameters & \multicolumn{1}{c}{ Meaning } & Value & Units \\
\hline$a$ & Normal macrophage influx & $5 \times 10^{4}$ & cells ml $^{-1} \mathrm{~d}^{-1}$ \\
$b$ & Recruitment rate of $M$ by $M_{a}$ & 0.09 & $\mathrm{~d}^{-1}$ \\
$c$ & Macrophage egress rate & 0.1 & $\mathrm{~d}^{-1}$ \\
$d$ & $B_{a}$ non-specific decay rate & 0.5 & $\mathrm{~d}^{-1}$ \\
$k$ & $M_{a}$ deactivation rate & 0.4 & $\mathrm{~d}^{-1}$ \\
$f_{1}$ & Basal phagocytosis rate per $M$ & $1 \times 10^{-5}$ & $\mathrm{ml} \mathrm{cell}^{-1} \mathrm{~d}^{-1}$ \\
$f_{2}$ & Activated phagocytosis rate per $M_{a}$ & $1 \times 10^{-5}$ & $\mathrm{ml} \mathrm{cell}^{-1} \mathrm{~d}^{-1}$ \\
$e$ & Anti-crowding rate & $2 \times 10^{-8}$ & $\mathrm{ml} \mathrm{cell}^{-1} \mathrm{~d}^{-1}$ \\
$A_{\max }$ & Maximal cytokine-induced $\beta$ cell apoptosis rate & cells ml m d $\mathrm{d}^{-1}$ \\
$k_{c}$ & Cytokine concentration for half-maximal apoptosis rate & 1.0 & $\mathrm{nM}^{7}$ \\
$\alpha$ & Cytokine secretion rate by $M_{a}$ due to $B_{n}$ & $5 \times 10^{-9}$ & $\mathrm{nM} \mathrm{cells}^{-2} \mathrm{~d}^{-1}$ \\
$\delta$ & Cytokine turnover rate & 25 & $\mathrm{~d}^{-1}$ \\
\hline
\end{tabular}

${ }^{\dagger}$ See (Marée et al., 2006) for references for the parameters.

\section{Appendix B. Brief description of the dynamics near the $f_{2}$-Hopf bifurcation}

In Fig. 4 we perturbed the system in the resting and activated macrophages and saw that at least one limit cycle encircles the disease state before it changes stability. Figure 4 only provided information about trajectories starting near $\mathbf{x}_{\mathrm{DS}}^{*}$. Here we present a bigger section of the phase space projection onto the $M-M_{a}$ phase plane (Fig. B1), but we also show what happens if we perturb the system in the $B_{a}, B_{n}$ and $C$ directions (Fig. B2), while using the $\mathbf{x}_{\mathrm{DS}}^{*}$-values of $M$ and $M_{a}$ as initial conditions for these variables. Figure B1 shows a bigger part of the $M-M_{a}$ phase plane for a range of perturbations; $P=\mathbf{x}_{\mathrm{DS}}^{*} \cdot(m / 2, n / 2,1,1,1)^{\mathrm{T}}, m=0, \ldots, 5, n=0, \ldots, 10$. A full dot indicates $\mathbf{x}_{H}^{*}$, while an open ring indicates $\mathbf{x}_{\mathrm{DS}}^{*}$. We have scaled everything relative to the disease state. It should be noted that It should be noted that the curves apparently tending to the disease state in fact approach the stable limit cycle encircling this state. 


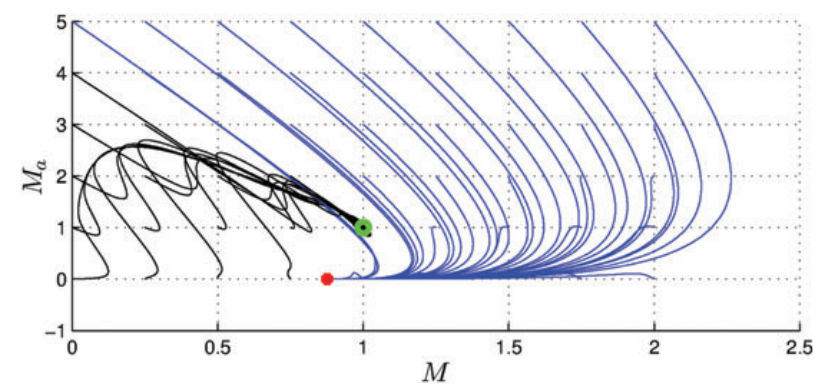

FIG. B1. Simulations, with $f_{2}=8.477652 \times 10^{-5}$, for a range of initial values plotted in the $M-M_{a}$ phase plane. The full dot is $\mathbf{x}_{H}^{*}$. The open ring indicates $\mathbf{x}_{\mathrm{DS}}^{*}$. Initial conditions for $B_{a}, B_{n}$ and $C$ were set to their $\mathbf{x}_{\mathrm{DS}}^{*}$-value. See Fig. B2 for legend.

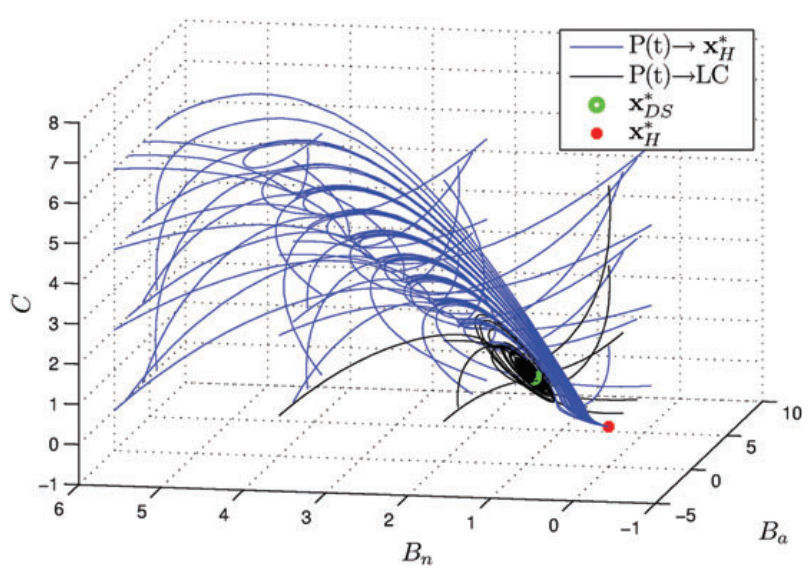

FIG. B2. Simulations, with $f_{2}=8.477652 \times 10^{-5}$, for a range of initial values plotted in the $B_{a}-B_{n}-C$ phase space. Initial conditions for $M$ and $M_{a}$ were set to their $\mathbf{x}_{\mathrm{DS}}^{*}$-value. $\mathbf{x}_{H}^{*}$ : healthy state; LC: limit cycle; $\mathbf{x}_{\mathrm{DS}}^{*}$ : disease state.

Perturbing the system in the $B_{a}, B_{n}$ and $C$ directions one at a time, without perturbing $M$ and $M_{a}$, gives some results that seem surprising at first. For small nudges away from $\mathbf{x}_{\mathrm{DS}}^{*}$ (small being relative to the values of the respective variables at the disease state) the state remains attracting, and stable spirals are observed-we can explain this by the negative feedback of $M_{a}$ (and $M$ ) on $B_{a}, B_{n}$ (through phagocytosis), as being dominant. As the perturbations grow, we eventually enter a domain where the stable limit cycle becomes attracting; see Fig. B2. Here the positive feedback, through $M_{a}$ 's participation in cytokine production, balances the negative feedbacks. Finally, in Fig. B2, we see that we have a 'fight fire with fire' situation; i.e. if we increase the initial values of $B_{a}, B_{n}$ and/or $C$ sufficiently, $\mathbf{x}_{H}^{*}$ becomes attracting. This is perhaps even more surprising than our suggested method of using macrophages to induce a return to the healthy state, but it can be explained by the extra inflow of macrophages that will occur when a large amount of resting macrophages suddenly become activated. However, inducing increased apoptosis, necrosis or injecting a bolus of proinflammatory cytokines, or a combination of these, does not seem like a viable approach to countering a burgeoning inflammation, simply because of the direct and collateral damage this will cause, which is not considered in the model.

If we decrease the initial values of $B_{a}, B_{n}$ or $C$ sufficiently, e.g. use $\mathbf{x}(0)=\mathbf{x}_{\mathrm{DS}}^{*} \cdot(1,1,0,1,1)^{\mathrm{T}}$, as initial condition, the system will tend to the stable limit cycle; see Fig. B2. (The same goes if we choose 
the dotproduct between $\mathbf{x}_{\mathrm{DS}}^{*}$ and $(1,1,1,0,1)^{\mathrm{T}}$ or $(1,1,1,1,0)^{\mathrm{T}}$ as initial condition.) The reason for this is that the concentration of nectrotic $\beta$ cells and cytokines (or $B_{a}$ and $C$ or $B_{a}$ and $B_{n}$ ) is sufficient to stabilize the positive feedback from $M_{a}$ on the 'harmful' variables.

When we decrease $f_{2}$, the basin of attraction in terms of $B_{a}, B_{n}$ and $C$ grows; e.g. for $f_{2}=7 \times$ $10^{-5}$ only two of the initial conditions seen in Fig. B2 tend to $\mathbf{x}_{H}^{*}$, namely $\mathbf{x}_{\mathrm{DS}} \cdot(1,1,0,0,0)^{\mathrm{T}}$ and $\mathbf{x}_{\mathrm{DS}} \cdot(1,1,6,6,6)^{\mathrm{T}}$-not shown. The explanation for this is that at lower $f_{2}$ values a larger number of activated macrophages need to enter the system to reach the same phagocytic capacity that was seen at higher $f_{2}$ values. Thus, at lower $f_{2}$ values we need to (instantaneously) supply a larger concentration of $B_{a}$ and/or $B_{n}$ and/or $C$ to get to that critical level of activated macrophages. As $f_{2}$ is increased, the disease state becomes unstable. However, it is a saddle point with four complex eigenvalues, the two of which have large negative real part compared with the other eigenvalues. Solution curves starting close to the unstable disease state for $f_{2}>f_{2, \text { hb }}$ oscillate for a period, which is much longer than the average lifespan of a laboratory mouse, before finally tending to $\mathbf{x}_{H}^{*}$. (Simulation not shown.) However, this pseudo-stability of the disease state is only observed for a very narrow window of values in $f_{2}$ and for initial conditions very close to $\mathbf{x}_{\mathrm{DS}}^{*}$, so we do not consider this a blow to our method of increasing $f_{2}$. 